\title{
A Fluid-Structure Coupling Method to Predict the Interior Ballistic Characteristic of Gas Generator with Complex Structures
}

\author{
Xinggan LU, Kun JIANG ${ }^{1}$, Shenshen CHENG and Hao WANG \\ Nanjing University of Science and Technology, Nanjing, 210094, China
}

\begin{abstract}
For gas generators based on pyrotechnic technology, as the structure becomes more complex, the internal ballistic characteristic is no longer just related to the flow field generated by propellant combustion. The fluid-structure interaction in the system plays an important role. In order to accurately predict the internal ballistic characteristic of the gas generator, a mathematical model of coupling the flow field generated by propellant combustion and the structural evolution in the system is established. The interior ballistic model is utilized to calculate the flow field generated by propellant combustion, and the finite element method is applied to simulate the structural evolution. The flow field and the structural evolution are coupled through a user subroutine interface in ABAQUS. The accuracy of the coupled model is verified by experiment, and the precision of the traditional classical interior ballistic model is compared with that of the coupled model. The results show that the pressure prediction error of the traditional classical interior ballistic model is more than $46 \%$ for gas generators with complex structures. While the coupling model is in great agreement with the experiment, and the error of pressure prediction is less than $5.0 \%$.
\end{abstract}

Keywords. Fluid-structure interaction, interior ballistic, gas generator.

\section{Introduction}

The gas generator based on pyrotechnic technology takes solid propellant as incendiary agent and oxidizer, which is filled in the chamber and ignited by the ignition device to generate high temperature and high pressure gas [1]. It is widely used in aerospace [2], ship [3], petrochemical [4] and automobile [5] and other fields. So far, researchers have done a lot of research on gas generators by means of theoretical analysis, experimental verification and numerical calculation. Great progress has been made in optimizing the structure, improving the performance and reliability, and reducing the cost [6-9].

With the application expansion of the gas generator, its requirements are becoming higher and higher. As a result, the structure and operating environment of gas generator become more and more complicated. And its interior ballistic characteristic can not only consider the influence of propellant combustion. Taj Wali Khan et al. [10] found that the characteristic speed and pressure of liquid rocket engine were strong functions of the characteristic length of gas generator, and obtained the optimal characteristic length. Salgansky E.A et al. [11] studied the performance of gas generators applied to high-speed

\footnotetext{
${ }^{1}$ Corresponding Author, Kun JIANG, Nanjing University of Science and Technology, Nanjing, 210094 , China; E-mail: 1332412141@qq.com.
} 
aircraft at low temperatures and analyzed the effect of fuel type. Afanasiev N. A. et al. [12] studied the problem of thermoacoustic instability in gas turbine combustor, and the propagation of long wave in aerodynamic channel is described by quasi-linear equations. Zhiyue Han et al. [13] studied a new flexible body deployment system for spacecraft and analyzed the performance of gas generator.

In this paper, a mathematical model that coupling flow field of propellant combustion and structural evolution is established for a gas generator with complex structures. The traditional classical interior ballistic model and the coupled model are used to simulate the corresponding interior ballistic characteristic. The simulation results and precision of the two models are compared.

\section{Gas Generator Structure Principle and Experiment}

\subsection{Structure Principle}

The structure diagram of gas generator studied in this paper is presented in figure 1, which mainly includes chamber, central sliding duct, burner, pistons and rubber cushion, and so on. The central sliding duct is positioned by shear pins. The vent holes are distributed in the wall of the burner along the axial direction, and each group is evenly distributed in the circumferential direction. And these holes are sealed by the pistons, which mounted on the central sliding duct, and their outer circumference is closely attached to the inner wall of the burner. The sides of middle pistons are opened with a plurality of the evenly distributed large diameter circular holes, the end piston side is not opened holes.

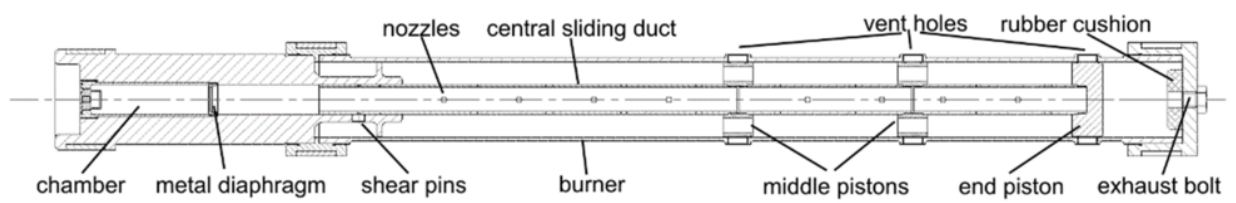

Figure 1. Structure diagram of gas generator.

The propellant is packed in the chamber. A metal diaphragm is used to seal chamber exits. As propellant is ignited and begins to burn, the metal diaphragm is sheared instantly when chamber pressure meets the set requirements, and the gas flows into the central sliding duct with the unburned propellant particles. Thereafter, the gas fills the space enclosed by the chamber, the central sliding duct, and the burner. The end piston receives axial thrust but remains fixed within shear pin constraints. Until the axial thrust increases to the point where the shear pin is severed, the central sliding duct and pistons begin to move. The vent holes are opened and the gas gradually flows out. When the end piston moves to the restricted position, the axial movement process ends and the gas generator enters a stable state.

\subsection{Experiment}

The gas generator used in the experiment is presented in figure 2. Piezoelectric sensors are used to obtain pressure data. A high-speed camera system is used to capture the 
timing sequence, brightness and shape of the flame at the vent hole outlet. The layout of the experiment site is presented in the figure 3.

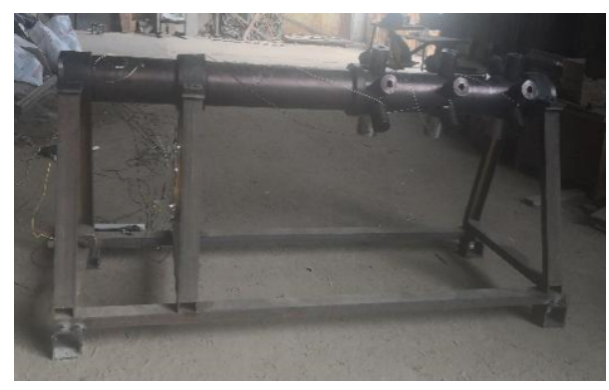

Figure 2. The gas generator used in experiment.

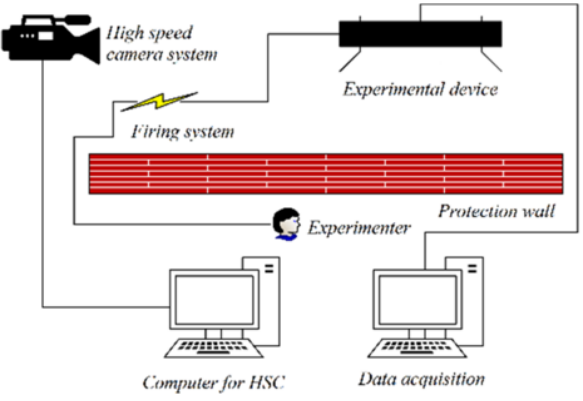

Figure 3. The experiment management.

Repeated experiments were carried out under different propellant mass conditions. Select a group the experiment with a propellant mass of $500 \mathrm{~g}$, the phenomenon of the opening moment of the vent holes is presented in the figure 4 . The pressure-time curve and the response time $T$ of the vent holes in the wall of the burner are obtained. The former is the main characteristic of the gas flowing out of the gas generator, while the latter represents the total time from the moment of ignition to the moment of opening the holes. When the propellant mass is $500 \mathrm{~g}$, the experimental value of $T$ is $6.4 \mathrm{~ms}$.

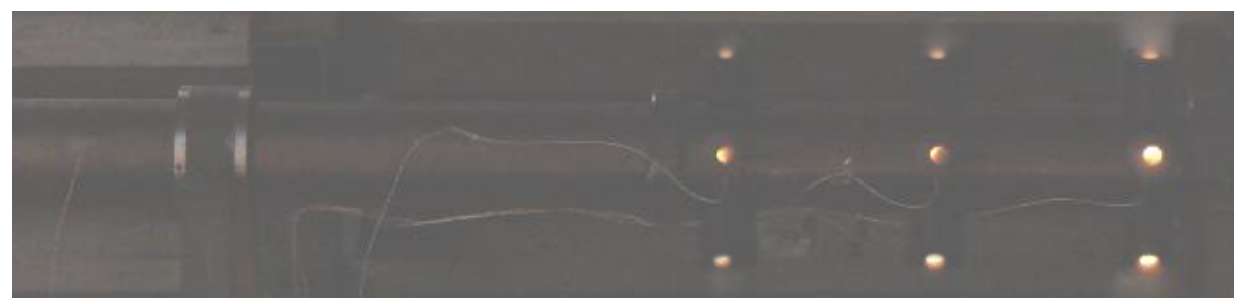

Figure 4. The opening moment of the vent holes.

The pressure at the burner will be used later to verify the accuracy of the model. And it should be noticed that the relevant parameters of propellant and gas generator used in the experiment are consistent with the data in the subsequent numerical simulation.

\section{Traditional Classical Interior Ballistic Model}

For the gas generator, the traditional classical interior ballistic model is usually used to obtain the characteristic, such as the pressure at the burner and the response time $T$. Although the model simplifies the interaction and energy dissipation in the system.

Based on the interior ballistic theory, the following assumptions are made:

(1)The combustion of propellant follows the geometrical combustion law and combustion velocity equation.

(2)The propellant gas obeys the Nobel equation.

(3)The composition of propellant and products is constant, and the propellant impetus, co-volume parameter and specific heat ratio are constant. 
(4)The combustion of the propellant and the movement of the duct and the pistons are carried out at an average pressure

(5)The flow of gas through the vent holes obeys the Bernoulli's principle.

The main internal ballistic equations are presented as equation (1). The detailed related formulas and the meaning of each symbol can be obtained from the reference [14], not to be repeated here.

$$
\begin{aligned}
& \begin{array}{r}
\psi=\chi Z\left(1+\lambda Z+\mu Z^{2}\right) \\
\frac{d Z}{d t}= \begin{cases}\frac{u_{1}}{e_{1}} p_{1}^{n}, Z<Z_{K} \\
0, & Z \geq Z_{K}\end{cases}
\end{array}
\end{aligned}
$$

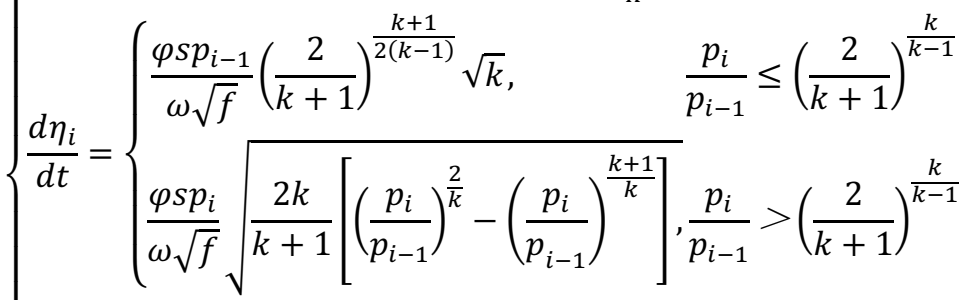

$$
\begin{aligned}
& p_{1}=\frac{f \omega\left(\psi-\eta_{2}\right)}{V_{1}-\frac{\omega}{\rho}(1-\psi)-\alpha \omega\left(\psi-\eta_{2}\right)} \\
& p_{i}=\frac{f \omega\left(\eta_{i}-\eta_{i+1}\right)}{V_{i}-\alpha \omega\left(\eta_{i}-\eta_{i+1}\right)}
\end{aligned}
$$

The fourth order Runge-Kutta method is used to calculate the program with above equations, and the internal ballistic characteristic of gas generator is numerically simulated. The pressure comparison in the burner between the simulation result of the traditional classical interior ballistic model and experimental result is presented in the figure 5 . The classical model only considers the relationship between the pressure and the combustion degree of the propellant, ignoring the interaction with structural evolution. The dissipated energy is still converted into gas pressure. Therefore, the two start in good agree, but diverge more and more over time. The max error is more than $46 \%$. In addition, the value of $T$ obtained by the classical model is $9.7 \mathrm{~ms}$, while being $6.4 \mathrm{~ms}$ in the experiment. Its error is $34 \%$.

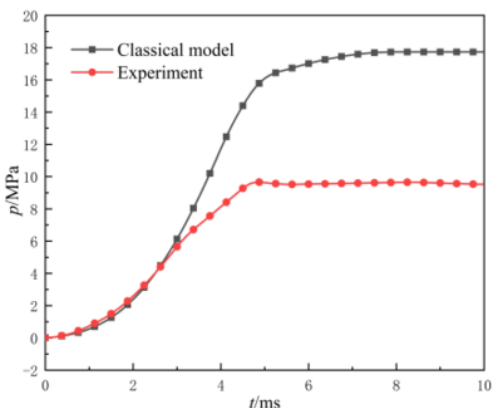

Figure 5. The pressure comparison in the burner.

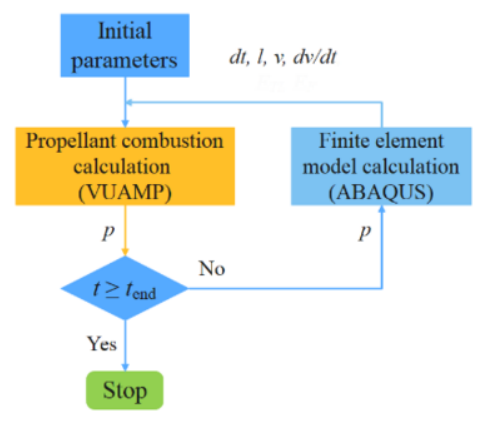

Figure 6. The coupling strategy. 
Obviously, the traditional classical interior ballistic model has no ability to accurately predict the internal ballistic characteristic of the gas generator with complex structures.

\section{Coupling Model}

The process of propellant combustion and gas flow in gas generator is regulated by four high and low pressure chambers and involves complex structural evolution, such as friction between components, movement of central sliding duct and pistons, compressed deformation of rubber cushion. All of these will in turn affect the flow field generated by propellant combustion.

In the traditional interior ballistic model, a constant coefficient is used to simplify the energy dissipation in the fluid-structure interaction. However, the friction dissipation is a function of the kinetic parameters of the moving body and the structural parameters of the system. And the strain energy of the rubber cushion cannot be described by the simple linear coefficients. Therefore, the traditional interior ballistic model is not capable of reflecting the fluid-structure interaction in the system.

In this paper, a numerical model of that couples flow field generated by the propellant combustion and the structural evolution of the system is provided, through a user subroutine interface VUAMP in ABAQUS. The coupling model is divided into two sub-systems. One considers the propellant combustion in VUAMP, and the other one considers the structural evolution in ABAQUS.

The fluid-structure interaction effect is complied by the interchange between load calculated from VUAMP and kinetic parameters obtained from ABAQUS, as presented in equation (2).

$$
M \ddot{U}+C \dot{U}+K U=Q
$$

Where $U, \dot{U}$ and $\ddot{U}$ are the displacement, velocity and acceleration vectors, respectively; $M, C$ and $K$ are the mass, damping and stiffness matrixes, respectively; and $Q$ is the loading vector.

The coupling strategy is presented in figure 6.

\subsection{Interior Ballistic Model}

Based on the classical interior ballistic model in Section 2, the equations governing the propellant combustion are programmed in FORTRAN in VUAMP. For each time step (about $1 \mathrm{~d}-7 \mathrm{~s}$ ) of the moving body advances, the VUAMP obtains the real-time kinetic parameters such as the displacement, velocity, acceleration from the finite element model in ABAQUS, and applies them for combustion calculation. In turn, the calculated pressure is conveyed to the actuator as the amplitude, and the next step is started. The finite element model adopts the updated load and then carries on the subsequent simulation. Until the coupling model meets the end criteria, the cycle calculation is complete. 


\subsection{Finite Element Model}

The gas generator structure is simplified, and only the central sliding duct, pistons, rubber cushion and part of the wall of the burner are considered. The corresponding finite element model is established in ABAQUS. Hexahedral elements are preferred for meshing. The grid size of the center sliding duct and pistons is $0.3 \mathrm{~mm}$, the grid size of the rubber cushion is $0.1 \mathrm{~mm}$, and the grid size of the burner is $1.0 \mathrm{~mm}$.

The material parameters of the above models are presented in table 1 . The central sliding duct, pistons and burner are made of carbon steel. The rubber cushion will use nitrile rubber.

Table 1. The relevant material parameters.

\begin{tabular}{cccccc}
\hline Material & Density $\left(\mathbf{k g ~ m}^{-\mathbf{3}}\right)$ & $\begin{array}{c}\text { Young's modulus } \\
(\mathbf{G P a})\end{array}$ & Poisson's ratio & $\mathbf{C}_{\mathbf{1 0}}$ (MPa) & $\mathbf{C}_{\mathbf{0 1}}$ (MPa) \\
\hline Carbon steel & 7800 & 210 & 0.3 & $\backslash$ & $\backslash$ \\
Nitrile rubber & 1000 & $\backslash$ & $\backslash$ & 1.9519 & -0.3067 \\
\hline
\end{tabular}

The phenomenological theory based on continuum mechanics assumes that rubber is isotropic material and its mechanical behavior is characterized by strain energy per unit volume $[15,16]$. In practical engineering applications, the results obtained by using Mooney-Rivlin model for rubber materials meet the engineering solving needs. This model supports most of the current technical units, especially the Mooney-Rivlin model by setting two parameters is more widely used to solve the problem. The expression of the two-parameter Mooney-Rivlin model is as follows in equation (3).

$$
W=C_{10}\left(I_{1}-3\right)+C_{01}\left(I_{2}-3\right)
$$

Where $W$ is the strain potential energy, $I_{1}$ is the first-order strain invariant, $I_{2}$ is the second-order strain invariant, and $\mathrm{C}_{10} 、 \mathrm{C}_{01}$ are Mooney constants.

In finite element analysis, the deformation of materials can be so intense that it is difficult to predict in advance where and how contact will take place. Therefore, the general contact options are chosen. And the friction formulation adopts the penalty function with the coefficient 0.25 .

\section{Result and Discussion}

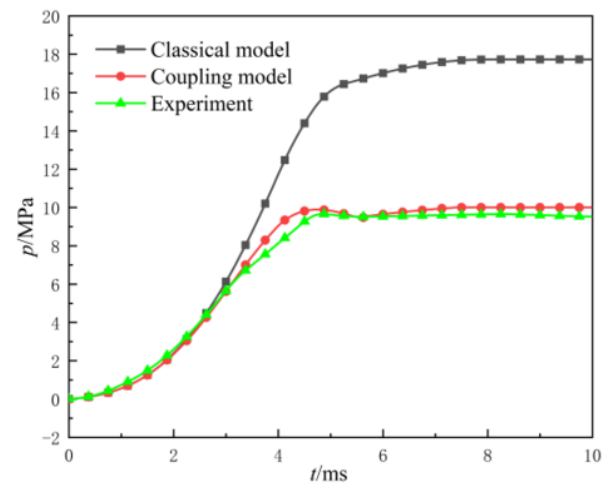

Figure 7. The comparison of pressure in the burner.

Table 2. The values of $T$.

\begin{tabular}{ccc}
\hline Source & $\begin{array}{c}\text { T } \\
(\mathbf{m s})\end{array}$ & $\begin{array}{c}\text { Error compared } \\
\text { with experiment }\end{array}$ \\
\hline $\begin{array}{c}\text { Experiment } \\
\text { Classical } \\
\text { model }\end{array}$ & 6.4 & $\backslash$ \\
$\begin{array}{c}\text { Coupled } \\
\text { model }\end{array}$ & 6.7 & $34 \%$ \\
\hline
\end{tabular}


The comparison of the pressure in the burner between simulation result of two models and experimental result is presented in the figure 7. It can be seen that the curve corresponding to coupling model is in great agree with the curve of the experiment. The error is less than $5.0 \%$.

In the figure 7, the pressure obtained from the experiment is slightly lower than the pressure of the coupled model. And in the later period, the pressure corresponding to the coupled model remains constant, while curve obtained from the experiment declines. This is because the simulation ignores the thermal loss in the experimental environment and the air leak of the device.

The values of $T$ corresponding to three sources are presented in table 2 . By error comparison, it is also proved that the coupled model is superior to the traditional classical interior ballistic model.

\section{Conclusion}

For gas generator with complex structures, a mathematical method is developed to couple the flow field generated by propellant combustion and the structural evolution in the system, through VUAMP, a subroutine interface in ABAQUS. With experimental verification, the coupled model is capable of accurately predicting the internal ballistic characteristic of gas generator. The error of predicted pressure is less than $5.0 \%$, and the error of $T$ is $3.1 \%$.

\section{References}

[1] Sarisin M, Ulas A and Tinaztepe T. Conceptual Design of a Connected Pipe Test Facility for Ramjet Applications[C]// Aiaa/asme/sae/asee Joint Propulsion Conference \& Exhibit. 2013.

[2] Pengnian Y et al. Direct-Connect Test of Solid Scramjet with Symmetrical Structure[J]. Energies, 2021, 14(17):5589-5589.

[3] Malinovsky I M, Nesterenko V G and Ivanov I G. Research and optimization of axial gas forces in turbines of turbojet bypass engines with afterburner combustion chamber[J]. Journal of Physics: Conference Series, 2021, 1925(1).

[4] Milković Marko et al. The Possibility of the Inert Gas Generator Environmental Operation Mode Utilization on Long Range 2 Oil Tankers During Discharge Operation[J]. NAŠE MORE: znanstveni časopis za more i pomorstvo, 2021, 68(2):131-136.

[5] Yurchenko et al. Design of the Airbag Inflation System Applicable to Conventional and Autonomous Vehicles[J]. Automotive Innovation, 2021, :1-10.

[6] Connaughton J W. Application of a hydrazine gas generator to vacuum ejector pumping of a chemical laser: AIAA-1977-0892[R]. Orlando, AIAA, 1977.

[7] Bown N, Darley M. Advanced Airbag Landing Systems for Planetary Landers[C]// 18th AIAA Aerodynamic Decelerator Systems Technology Conference and Seminar. 2005.

[8] Vyas, Engblom W A, Georgiadis N J et al. Numerical Simulation of Vitiation Effects on a HydrogenFueled Dual-Mode Scramjet[R]. Orlando:AIAA, 2010.

[9] D Vaughn, D Garrard. Transitioning to Fly the Mission[C]// U.S. Air Force T\&E Days 2010. 2010.

[10] Taj Wali Khan and Ihtzaz Qamar. Optimum characteristic length of gas generator for liquid propellant rocket engine[J]. Acta Astronautica, 2020, 176:1-12.

[11] Salgansky E.A. and Lutsenko N.A.. Effect of solid fuel characteristics on operating conditions of lowtemperature gas generator for high-speed flying vehicle[J]. Aerospace Science and Technology, 2021, 109.

[12] Afanasiev N A et al. Direct Simulation of Thermoacoustic Instability in Gas Generators Using the CABARET Scheme[J]. Mathematical Models and Computer Simulations, 2021, 13(5):820-830.

[13] Zhiyue Han et al. A novel of spacecraft flexible compartment safe and stable inflatable expansion system with the environmental-friendly fuel[J]. Journal of Cleaner Production, 2021, 279. 
[14] Zhiming J. Interior ballistics of guns[M]. Beijing: Beijing Institute of Technology Press, 2004.(in Chinese).

[15] LU Chong, Bo Dong, Yu Cao. Analysis and Research on Rubber Joint of the Axle Box Rotary Arm of CRH3 EMU[J]. IOP Conference Series: Earth and Environmental Science, 2019, 267(4).

[16] Arya, Sarangi, Bhattacharyya. A damaged Ogden material tube under pressure: stability and bifurcation analysis[J]. Soft Materials, 2020, 18(1):74-88. 